\title{
Petiolins F-I, Benzophenone Rhamnosides from Hypericum pseudopetiolatum var. kiusianum
}

\author{
Naonobu Tanaka, ${ }^{a}$ Takaaki Kubota, ${ }^{a}$ Yoshiki Kashiwada, ${ }^{b}$ Yoshihisa TaKaishi, ${ }^{b}$ and \\ Jun'ichi KoBAYASHI $*, a$ \\ ${ }^{a}$ Graduate School of Pharmaceutical Sciences, Hokkaido University; Sapporo 060-0812, Japan: and ${ }^{b}$ Graduate School of \\ Pharmaceutical Sciences, University of Tokushima; Tokushima 770-8505, Japan.
}

Received June 29, 2009; accepted July 27, 2009; published online July 28, 2009

Four new benzophenone-O-rhamnosides, petiolins F-I (1-4), were isolated from aerial parts of Hypericum pseudopetiolatum var. kiusianum, and the structures were elucidated by spectroscopic data and chemical means.

Key words Hypericum pseudopetiolatum var. kiusianum; benzophenone; rhamnoside; Clusiaceae

The genus Hypericum (family Clusiaceae) are known to be a traditional medicine for the treatment of burns, bruises, swelling, inflammation, and anxiety as well as bacterial and viral infections. ${ }^{1-4)}$ In our continuing search for new compounds from Hypericum spp., ${ }^{5-7)}$ four new benzophenone$O$-rhamnosides, petiolins $\mathrm{F}$ - I $(\mathbf{1}-\mathbf{4})$, were isolated from aerial parts of $H$. pseudopetiolatum var. kiusianum. In this paper, we describe the isolation and structure elucidation of petiolins F-I (1-4).

The aerial parts of $H$. pseudopetiolatum var. kiusianum were extracted with $\mathrm{MeOH}$, and the extracts were partitioned successively with $n$-hexane, EtOAc, and $\mathrm{H}_{2} \mathrm{O}$. EtOAc-soluble portions were subjected to a Sephadex LH-20 column $\left(\mathrm{H}_{2} \mathrm{O} / \mathrm{MeOH}\right)$, a Toyopearl HW-40F column $\left(\mathrm{H}_{2} \mathrm{O} / \mathrm{MeOH}\right)$, and a silica gel column $\left(\mathrm{CHCl}_{3} / \mathrm{MeOH}\right)$ chromatographies to afford a mixture of benzophenone glycosides, which was purified by $\mathrm{C}_{18}$ HPLC $\left(\mathrm{MeOH} / \mathrm{H}_{2} \mathrm{O}\right)$ to yield petiolins $\mathrm{F}$ (1, $0.0012 \%), \mathrm{G}(\mathbf{2}, 0.0038 \%), \mathrm{H}(\mathbf{3}, 0.0006 \%)$, and I (4, $0.0004 \%)$.

The molecular formula of petiolin $\mathrm{F}(\mathbf{1}), \mathrm{C}_{19} \mathrm{H}_{20} \mathrm{O}_{10}$, was established by HR-electrospray ionization (ESI)-MS $[\mathrm{m} / \mathrm{z}$ $431.0945(\mathrm{M}+\mathrm{Na})^{+}, \Delta-0.9 \mathrm{mmu}$. IR absorptions at 3421 and $1629 \mathrm{~cm}^{-1}$ implied the presence of hydroxy and carbonyl functionalities. The ${ }^{1} \mathrm{H}-\mathrm{NMR}$ spectrum showed proton signals of a 1,3,5-trisubstituted benzene ring $\left[\delta_{\mathrm{H}} 6.58(2 \mathrm{H}, \mathrm{d}\right.$, $J=2.3 \mathrm{~Hz}), 6.51(1 \mathrm{H}, \mathrm{t}, J=2.3 \mathrm{~Hz})]$, a 1,2,3,5-tetrasubstituted benzene ring $\left[\delta_{\mathrm{H}} 6.37\right.$ and $6.12(1 \mathrm{H}$ each, d, $\left.J=2.0 \mathrm{~Hz})\right]$, an anomeric proton $\left[\delta_{\mathrm{H}} 5.26(1 \mathrm{H}, \mathrm{d}, J=1.5 \mathrm{~Hz})\right]$, and a secondary methyl group $[1.16(3 \mathrm{H}, \mathrm{d}, J=6.3 \mathrm{~Hz})]$ (Table 1$)$. The ${ }^{13} \mathrm{C}$-NMR spectrum revealed the presence of a carbonyl $\left(\delta_{\mathrm{C}}\right.$ 198.2) and 12 aromatic carbons, together with resonances for
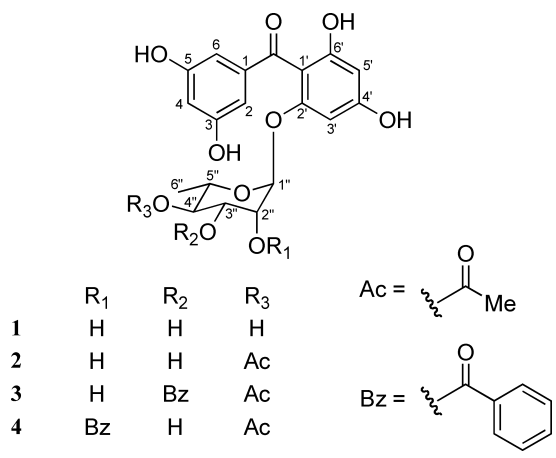

Chart 1. Petiolins F-I (1-4) a sugar moiety (Table 1). From these data, 1 was presumed to be a benzophenone glycoside. ${ }^{13} \mathrm{C}$-NMR chemical shifts of the sugar moiety were coincident with those of quercetin3-O- $\alpha$-rhamnoside. ${ }^{8)}$ The aglycone of $\mathbf{1}$ was assigned as $2^{\prime}, 3,4^{\prime}, 5,6^{\prime}$-pentahydroxybenzophenone on the basis of heteronuclear multiple bond correlations (HMBC) (Fig. 1) and coupling patterns of aromatic protons in the ${ }^{1} \mathrm{H}-\mathrm{NMR}$ (Table 1). The HMBC correlation for $\mathrm{H}-1^{\prime \prime}$ to $\mathrm{C}-2^{\prime}$ indicated that the rhamnosyl moiety was connected to $\mathrm{C}-2^{\prime}$ through an oxygen atom, and its $\alpha$-glycoside linkage was derived from the value for ${ }^{1} J_{\mathrm{C}, \mathrm{H}}(172 \mathrm{~Hz})$ of C-1" obtained from the non-decoupled heteronuclear single quantum coherence (HSQC) spectrum. ${ }^{9)}$ Methanolysis of petiolin F (1) yielded methyl $\alpha$-rhamnopyranoside, which was assigned as L-form by comparison of its optical rotation with that of authentic methyl $\alpha$-L-rhamnopyranoside. Thus, the structure of $\mathbf{1}$ was elucidated to be $2^{\prime}, 3,4^{\prime}, 5,6^{\prime}$-pentahydroxybenzophenone- $2^{\prime}-O-\alpha$-L-rhamnoside.

Petiolin G (2) showed the pseudomolecular ion peak at $\mathrm{m} / \mathrm{z}$ $473(\mathrm{M}+\mathrm{Na})^{+}$in the ESI-MS, and the HR-ESI-MS revealed the molecular formula to be $\mathrm{C}_{21} \mathrm{H}_{22} \mathrm{O}_{11}$. Although ${ }^{1} \mathrm{H}$ - and

Table 1. ${ }^{1} \mathrm{H}$ - and ${ }^{13} \mathrm{C}-\mathrm{NMR}$ Data for Petiolins F (1) and G (2) in Acetone$d_{6}$

\begin{tabular}{|c|c|c|c|c|}
\hline \multirow{2}{*}{ Position } & \multicolumn{2}{|r|}{1} & \multicolumn{2}{|r|}{2} \\
\hline & ${ }^{13} \mathrm{C}$ & ${ }^{1} \mathrm{H}$ & ${ }^{13} \mathrm{C}$ & ${ }^{1} \mathrm{H}$ \\
\hline 1 & 143.9 & - & 143.8 & - \\
\hline 2,6 & 106.7 & $6.58(2 \mathrm{H}, \mathrm{d}, J=2.3)$ & 106.4 & $6.59(2 \mathrm{H}, \mathrm{d}, J=2.2)$ \\
\hline 3,5 & 158.5 & - & 158.6 & - \\
\hline 4 & 106.1 & $6.51(1 \mathrm{H}, \mathrm{t}, J=2.3)$ & 106.0 & $6.52(1 \mathrm{H}, \mathrm{d}, J=2.2)$ \\
\hline $1^{\prime}$ & 106.8 & - & 106.8 & - \\
\hline $2^{\prime}$ & 159.3 & - & 158.9 & - \\
\hline $3^{\prime}$ & 94.8 & $6.37(1 \mathrm{H}, \mathrm{d}, J=2.0)$ & 94.4 & $6.33(1 \mathrm{H}, \mathrm{d}, J=2.1)$ \\
\hline $4^{\prime}$ & 163.9 & - & 163.8 & - \\
\hline $5^{\prime}$ & 97.0 & $6.12(1 \mathrm{H}, \mathrm{d}, J=2.0)$ & 97.0 & $6.13(1 \mathrm{H}, \mathrm{d}, J=2.1)$ \\
\hline $6^{\prime}$ & 163.1 & - & 163.1 & - \\
\hline $\mathrm{C}=\mathrm{O}$ & 198.2 & - & 198.0 & - \\
\hline $1^{\prime \prime}$ & 99.5 & $5.26(1 \mathrm{H}, \mathrm{d}, J=1.5)$ & 98.9 & $5.33(1 \mathrm{H}, \mathrm{brs})$ \\
\hline $2^{\prime \prime}$ & 70.4 & $3.39(1 \mathrm{H}$, br s $)$ & 70.3 & $3.51(1 \mathrm{H}, \mathrm{brs})$ \\
\hline $3^{\prime \prime}$ & 71.2 & $3.00(1 \mathrm{H}, \mathrm{dd}, J=9.3,3.5)$ & 68.7 & $3.08(1 \mathrm{H}, \mathrm{dd}, J=9.8,3.4)$ \\
\hline $4^{\prime \prime}$ & 72.8 & $3.30(1 \mathrm{H}, \mathrm{t}, J=9.3)$ & 73.9 & $4.79(1 \mathrm{H}, \mathrm{t}, J=9.8)$ \\
\hline $5^{\prime \prime}$ & 69.9 & $3.42(1 \mathrm{H}, \mathrm{dq}, J=9.3,6.3)$ & 67.7 & $3.53(1 \mathrm{H}, \mathrm{dq}, J=9.8,6.4)$ \\
\hline $6^{\prime \prime}$ & 17.6 & $1.16(3 \mathrm{H}, \mathrm{d}, J=6.3)$ & 17.3 & $1.03(3 \mathrm{H}, \mathrm{d}, J=6.4)$ \\
\hline \multirow[t]{2}{*}{$-\mathrm{OAc}$} & - & - & 170.4 & - \\
\hline & & & 20.3 & $2.02(3 \mathrm{H}, \mathrm{s})$ \\
\hline
\end{tabular}

Coupling constants given $(J, \mathrm{~Hz})$ in parentheses. 
${ }^{13} \mathrm{C}$-NMR resonances of $\mathbf{2}$ were similar to those of $\mathbf{1}$, additional signals due to an acetoxy group $\left[\delta_{\mathrm{H}} 2.02(3 \mathrm{H}, \mathrm{s}) ; \delta_{\mathrm{C}}\right.$ 170.4 and 20.3] were observed in $\mathbf{2}$ (Table 1). From these data, 2 was estimated to be $2^{\prime}, 3,4^{\prime}, 5,6^{\prime}$-pentahydroxybenzophenone- $2^{\prime}-O$ - $\alpha$-rhamnoside possessing an acetoxy group. The HMBC cross-peak of $\mathrm{H}-4^{\prime \prime}$ to acetoxy carbonyl carbon and a low-field shift of H-4" ( $\delta_{\mathrm{H}} 4.79$ in $2 ; \delta_{\mathrm{H}} 3.30$ in 1$)$ indicated that the acetoxy group was connected to C-4". The rhamnose moiety was assigned as L-form by the same procedure as described for $\mathbf{1}$. Thus, the structure of $\mathbf{2}$ was assigned as $2^{\prime}, 3,4^{\prime}, 5,6^{\prime}$-pentahydroxybenzophenone- $2^{\prime}-O-\left(4^{\prime \prime}\right.$ acetoxy)- $\alpha$-L-rhamnoside.

Petiolin $\mathrm{H}$ (3) had a molecular formula of $\mathrm{C}_{28} \mathrm{H}_{26} \mathrm{O}_{12}$ deduced from HR-ESI-MS. The ${ }^{1} \mathrm{H}$ - and ${ }^{13} \mathrm{C}-\mathrm{NMR}$ data (Table 2) revealed the presence of a $2^{\prime}, 3,4^{\prime}, 5,6^{\prime}$-pentahydroxybenzophenone moiety, an acetoxy group, a benzoyl group, and a rhamnosyl moiety. Connectivities of the acetoxy group and the benzoyl group to the rhamnosyl moiety were elucidated by HMBC correlations for H-3" to the carbonyl carbon of the benzoyl group $\left(\delta_{\mathrm{C}} 165.0\right)$, and $\mathrm{H}-4^{\prime \prime}$ to acetoxy carbonyl carbon $\left(\delta_{\mathrm{C}} 170.0\right)$, respectively. The HMBC cross-peak of H-1" to $\mathrm{C}-2^{\prime}$ and ${ }^{1} J_{\mathrm{C}, \mathrm{H}}$ value $(174 \mathrm{~Hz})$ of $\mathrm{C}-1^{\prime \prime}$ indicated the connectivity of $\mathrm{C}-2^{\prime}$ and $\mathrm{C}-1^{\prime \prime}$ by an $\alpha$-glycoside linkage. The

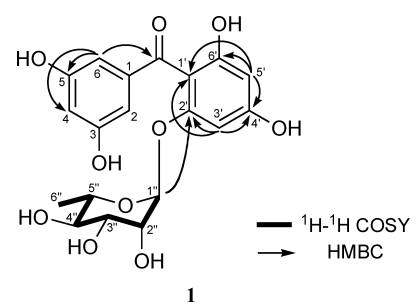

Fig. 1. Selected 2D NMR Correlations for Petiolin F (1)

Table 2. ${ }^{1} \mathrm{H}-$ and ${ }^{13} \mathrm{C}-\mathrm{NMR}$ Data for Petiolins H (3) and I (4) in Acetone$d_{6}$

\begin{tabular}{|c|c|c|c|c|}
\hline \multirow{2}{*}{ Position } & \multicolumn{2}{|r|}{3} & \multicolumn{2}{|r|}{4} \\
\hline & ${ }^{13} \mathrm{C}$ & ${ }^{1} \mathrm{H}$ & ${ }^{13} \mathrm{C}$ & ${ }^{1} \mathrm{H}$ \\
\hline 1 & 143.5 & - & 143.8 & - \\
\hline 2,6 & 107.0 & $6.68(2 \mathrm{H}, \mathrm{d}, J=2.3)$ & 106.5 & $6.66(2 \mathrm{H}, \mathrm{d}, J=2.2)$ \\
\hline 3,5 & 158.6 & - & 158.9 & - \\
\hline 4 & 106.4 & $6.46(1 \mathrm{H}, \mathrm{t}, J=2.3)$ & 106.3 & $6.58(1 \mathrm{H}, \mathrm{t}, J=2.2)$ \\
\hline $1^{\prime}$ & 107.6 & - & 106.3 & - \\
\hline $2^{\prime}$ & 158.8 & - & 158.6 & - \\
\hline $3^{\prime}$ & 94.7 & $6.38(1 \mathrm{H}, \mathrm{d}, J=2.0)$ & 94.8 & $6.34(1 \mathrm{H}, \mathrm{d}, J=2.0)$ \\
\hline $4^{\prime}$ & 163.9 & - & 164.4 & - \\
\hline $5^{\prime}$ & 97.2 & $6.17(1 \mathrm{H}, \mathrm{d}, J=2.0)$ & 97.6 & $6.17(1 \mathrm{H}, \mathrm{d}, J=2.0)$ \\
\hline $6^{\prime}$ & 163.4 & - & 163.8 & - \\
\hline $\mathrm{C}=\mathrm{O}$ & 197.8 & - & 197.9 & - \\
\hline $1^{\prime \prime}$ & 99.3 & $5.41(1 \mathrm{H}, \mathrm{d}, J=1.8)$ & 96.2 & $5.57(1 \mathrm{H}, \mathrm{d}, J=1.6)$ \\
\hline $2^{\prime \prime}$ & 68.0 & $3.99(1 \mathrm{H}, \mathrm{brs})$ & 72.8 & $5.07(1 \mathrm{H}, \mathrm{dd}, J=3.6,1.6)$ \\
\hline $3^{\prime \prime}$ & 72.9 & $4.59(1 \mathrm{H}, \mathrm{dd}, J=10.1,3.2)$ & 67.1 & $3.87(1 \mathrm{H}, \mathrm{dd}, J=9.9,3.6)$ \\
\hline $4^{\prime \prime}$ & 70.6 & $5.24(1 \mathrm{H}, \mathrm{t}, J=10.1)$ & 74.1 & $4.96(1 \mathrm{H}, \mathrm{t}, J=9.9)$ \\
\hline $5^{\prime \prime}$ & 68.0 & $3.72(1 \mathrm{H}, \mathrm{dq}, J=10.1,6.4)$ & 68.2 & $3.63(1 \mathrm{H}, \mathrm{dq}, J=9.9,6.4)$ \\
\hline $6^{\prime \prime}$ & 17.4 & $1.12(3 \mathrm{H}, \mathrm{d}, J=6.4)$ & 17.6 & $1.11(3 \mathrm{H}, \mathrm{d}, J=6.4)$ \\
\hline \multirow[t]{2}{*}{$-\mathrm{OAc}$} & 170.0 & - & 170.4 & - \\
\hline & 20.2 & $1.96(3 \mathrm{H}, \mathrm{s})$ & 20.5 & $2.08(3 \mathrm{H}, \mathrm{s})$ \\
\hline \multirow[t]{5}{*}{$-\mathrm{OBz}$} & 165.0 & - & 165.7 & - \\
\hline & 130.7 & - & 130.4 & - \\
\hline & 133.3 & $7.62(1 \mathrm{H}, \mathrm{tt}, J=8.4,1.3)$ & 133.7 & $7.66(1 \mathrm{H}, \mathrm{tt}, J=7.8,1.9)$ \\
\hline & $129.9 \times 2$ & $7.49(2 \mathrm{H}, \mathrm{t}, J=8.4)$ & $130.0 \times 2$ & $7.54(2 \mathrm{H}, \mathrm{t}, J=7.8)$ \\
\hline & $128.7 \times 2$ & $7.94(2 \mathrm{H}, \mathrm{dd}, J=8.4,1.3)$ & $128.9 \times 2$ & $8.05(2 \mathrm{H}, \mathrm{dd}, J=7.8,1.9)$ \\
\hline
\end{tabular}

Coupling constants given $(J, \mathrm{~Hz})$ in parentheses. rhamnose moiety was elucidated to be L-form in the same manner as described for $\mathbf{1}$. Thus, the structure of $\mathbf{3}$ was elucidated to be $2^{\prime}, 3,4^{\prime}, 5,6^{\prime}$-pentahydroxybenzophenone-(4'-acetoxy-3"-benzoyl)- $O$ - $\alpha$-L-rhamnoside.

Petiolin I (4) had the same molecular formula as that of 3. The ${ }^{1} \mathrm{H}$ - and ${ }^{13} \mathrm{C}-\mathrm{NMR}$ spectral data of 4 (Table 2) revealed the presence of the same functional groups as found in $\mathbf{3}$, while differences were observed for the proton resonances for the rhamnosyl moiety. The chemical shifts of $\mathrm{H}-2^{\prime \prime}\left(\delta_{\mathrm{H}}\right.$ 5.07) and $\mathrm{H}-4^{\prime \prime}\left(\delta_{\mathrm{H}} 4.96\right)$ suggested that a benzoyl and an acetoxy groups were attached to $\mathrm{C}-2^{\prime \prime}$ and $\mathrm{C}-4$ ", respectively. Positions of the acetoxy group and the benzoyl group were assigned as C-4" and C-2", respectively, by the HMBC crosspeaks of H-4" to the acetoxy carbonyl carbon $\left(\delta_{\mathrm{C}} 170.4\right)$ and $\mathrm{H}-2^{\prime \prime}$ to the benzoyl carbonyl carbon $\left(\delta_{\mathrm{C}} 165.7\right)$. The L-form of rhamnose moiety of $\mathbf{4}$ was assigned by the same procedure as described for $\mathbf{1}$. Thus, the structure of $\mathbf{4}$ was elucidated to be $2^{\prime}, 3,4^{\prime}, 5,6^{\prime}$-pentahydroxybenzophenone-(4"-acetoxy$2^{\prime \prime}$-benzoyl)- $O$ - $\alpha$-L-rhamnoside.

\section{Experimental}

General Optical rotations were recorded on a JASCO P-1030 digital polarimeter. IR and UV spectra were recorded on JASCO FT/IR-230 and Shimadzu UV-1600PC spectrophotometers, respectively. NMR spectra were measured with a JEOL ECA 500 spectrometer. The 2.05 and $205.7 \mathrm{ppm}$ resonances of residual acetone were used as internal references for ${ }^{1} \mathrm{H}$ - and ${ }^{13} \mathrm{C}$ NMR spectra, respectively. ESI-MS spectra were recorded on a JEOL JMST100LP.

Plant Material Hypericum pseudopetiolatum var. kiusianum was collected in Kochi Prefecture, Japan in August 2005. Herbarium specimens were deposited in the botanical garden of the University of Tokushima (specimen number: UTP98013)

Extraction and Isolation The aerial parts of $H$. pseudopetiolatum var. kiusianum $(320 \mathrm{~g})$ were extracted with $\mathrm{MeOH}(31 \times 3)$, and the extracts were partitioned successively with $n$-hexane $(300 \mathrm{ml} \times 3)$, EtOAc $(300 \mathrm{ml} \times 3)$, and $\mathrm{H}_{2} \mathrm{O}(300 \mathrm{ml})$. The EtOAc-soluble portions were subjected to a Sephadex LH-20 column $\left(\mathrm{H}_{2} \mathrm{O} / \mathrm{MeOH}, 10 / 0\right.$ to $\left.0 / 10\right)$, a Toyopearl HW-40F column $\left(\mathrm{H}_{2} \mathrm{O} / \mathrm{MeOH}, 9 / 1\right.$ to $\left.0 / 10\right)$, a silica gel column $\left(\mathrm{CHCl}_{3} / \mathrm{MeOH}, 95 / 5\right.$ to $\left.0 / 10\right)$, and $\mathrm{C}_{18}$ reversed-phase HPLC (Mighty sil RP-18, Kanto Chemical Co., Ltd., $10 \times 250 \mathrm{~mm}$; flow rate $3.0 \mathrm{ml} / \mathrm{min}$; UV detection at $254 \mathrm{~nm}$; eluent $\left.\mathrm{MeOH} / \mathrm{H}_{2} \mathrm{O}, 3: 7\right)$ to afford petiolins $\mathrm{F}-\mathrm{I}(\mathbf{1}, 3.9 \mathrm{mg} ; \mathbf{2}, 12.1 \mathrm{mg} ; \mathbf{3}, 1.9 \mathrm{mg}$; 4, $1.4 \mathrm{mg})$.

Petiolin F (1): Colorless amorphous solids; $[\alpha]_{\mathrm{D}}^{23}+5.8(c=0.87 \mathrm{MeOH})$; $\mathrm{UV}(\mathrm{MeOH}) \lambda_{\max } 280(\varepsilon 4650)$ and $307(5730) \mathrm{nm}$; IR (KBr) $v_{\max } 3421$ and $1629 \mathrm{~cm}^{-1}$; ${ }^{1} \mathrm{H}$ - and ${ }^{13} \mathrm{C}-\mathrm{NMR}$ data (Table 1); ESI-MS $m / z$ : $431(\mathrm{M}+\mathrm{Na})^{+}$; HR-ESI-MS $m / z$ : $431.0945(\mathrm{M}+\mathrm{Na})^{+}$(Calcd for $\left.\mathrm{C}_{19} \mathrm{H}_{20} \mathrm{O}_{10} \mathrm{Na}, 431.0954\right)$.

Petiolin G (2): Colorless amorphous solids; $[\alpha]_{\mathrm{D}}^{23}-4.9(c=2.45 \mathrm{MeOH})$; UV (MeOH) $\lambda_{\max } 277$ ( $\varepsilon$ 6920) and $308(8340) \mathrm{nm}$; IR (KBr) $v_{\max } 3407$, 1723, and $1627 \mathrm{~cm}^{-1} ;{ }^{1} \mathrm{H}$ - and ${ }^{13} \mathrm{C}$-NMR data (Table 1); ESI-MS $\mathrm{m} / \mathrm{z}$ : 473 $(\mathrm{M}+\mathrm{Na})^{+}$; HR-ESI-MS $m / z$ : $473.1048(\mathrm{M}+\mathrm{Na})^{+}\left(\mathrm{Calcd}\right.$ for $\mathrm{C}_{21} \mathrm{H}_{22} \mathrm{O}_{11} \mathrm{Na}$, 473.1060).

Petiolin H (3): Colorless amorphous solids; $[\alpha]_{\mathrm{D}}^{23}-54.0(c=0.38 \mathrm{MeOH})$; $\mathrm{UV}(\mathrm{MeOH}) \lambda_{\max } 281\left(\varepsilon\right.$ 8660) and $306(8800) \mathrm{nm}$; IR (KBr) $v_{\max } 3417$, 1723, and $1627 \mathrm{~cm}^{-1}$; ${ }^{1} \mathrm{H}$ - and ${ }^{13} \mathrm{C}-\mathrm{NMR}$ data (Table 2); ESI-MS $\mathrm{m} / z$ : 577 $(\mathrm{M}+\mathrm{Na})^{+}$; HR-ESI-MS m/z: $577.1323(\mathrm{M}+\mathrm{Na})^{+}\left(\mathrm{Calcd}\right.$ for $\mathrm{C}_{28} \mathrm{H}_{26} \mathrm{O}_{12} \mathrm{Na}$, 577.1322).

Petiolin I (4): Colorless amorphous solids; $[\alpha]_{\mathrm{D}}^{23}-19.2(c=0.27 \mathrm{MeOH})$; $\mathrm{UV}(\mathrm{MeOH}) \lambda_{\max } 275(\varepsilon 8880)$ and $305(8070) \mathrm{nm}$; IR (KBr) $v_{\max } 3442$, 1727, and $1619 \mathrm{~cm}^{-1} ;{ }^{1} \mathrm{H}$ - and ${ }^{13} \mathrm{C}-\mathrm{NMR}$ data (Table 2); ESI-MS $\mathrm{m} / z$ : 577 $(\mathrm{M}+\mathrm{Na})^{+}$; HR-ESI-MS $m / z$ : $577.1331(\mathrm{M}+\mathrm{Na})^{+}\left(\right.$Calcd for $\mathrm{C}_{28} \mathrm{H}_{26} \mathrm{O}_{12} \mathrm{Na}$, 577.1322).

Methanolysis of Petiolins F-I (1-4) Petiolins F- I (1-4, 0.7, 0.7, 0.5 , and $0.5 \mathrm{mg}$, respectively) were treated with $5 \% \mathrm{HCl} / \mathrm{MeOH}(50 \mathrm{ml})$ at $100^{\circ} \mathrm{C}$ for $16 \mathrm{~h}$, individually. After evaporation of the solvent, the residue of each sample was subjected to a silica gel column (EtOAc/ $\mathrm{MeOH} / \mathrm{H}_{2} \mathrm{O}$, $20: 3: 2$ ) to give methyl $\alpha$-rhamnopyranoside from 1: $0.13 \mathrm{mg},[\alpha]_{\mathrm{D}}^{23}-67.9$ $(c=0.03, \mathrm{MeOH})$; from 2: $0.22 \mathrm{mg},[\alpha]_{\mathrm{D}}^{23}-61.0(c=0.08, \mathrm{MeOH})$; from 3: $0.15 \mathrm{mg},[\alpha]_{\mathrm{D}}^{23}-71.4(c=0.04, \mathrm{MeOH})$; from $4: 0.17 \mathrm{mg},[\alpha]_{\mathrm{D}}^{23}-74.4$ $(c=0.04, \mathrm{MeOH})\}$ and $2^{\prime}, 3,4^{\prime}, 5,6^{\prime}$-pentahydroxybenzophenone. $2^{\prime}, 3,4^{\prime}, 5$, $6^{\prime}$-Pentahydroxybenzophenone: ${ }^{1} \mathrm{H}-\mathrm{NMR}$ (acetone- $\left.d_{6}\right) \delta_{\mathrm{H}}: 6.59(2 \mathrm{H}, \mathrm{d}$, 
$J=1.6 \mathrm{~Hz}), 6.47(1 \mathrm{H}, \mathrm{t}, J=1.6 \mathrm{~Hz}), 5.96(2 \mathrm{H}, \mathrm{s})$; HR-ESI-MS $m / z: 285.0382$ $(\mathrm{M}+\mathrm{Na})^{+}\left(\right.$Calcd for $\left.\mathrm{C}_{13} \mathrm{H}_{10} \mathrm{O}_{6} \mathrm{Na}, 285.0375\right)$. Authentic L-rhamnose was treated with $5 \% \mathrm{HCl} / \mathrm{MeOH}$ as described above to afford methyl $\alpha$-Lrhamnopyranoside $\left\{[\alpha]_{\mathrm{D}}-64.8(c=0.19, \mathrm{MeOH})\right\}$. $R f$ values of methyl $\alpha$ L-rhamnopyranosides derived from $1-4$ were consistent with that of authentic methyl $\alpha$-L-rhamnopyranoside ( $R f$ value: 0.66 , silica gel TLC, EtOAc/ $\mathrm{MeOH} / \mathrm{H}_{2} \mathrm{O}, 20: 3: 2$ ).

Acknowledgements We thank T. Akiyama and M. Inagaki, the Kochi Prefectural Makino Botanical Garden, for collection and botanical identification of the plant, S. Oka and A. Tokumitsu, Center for Instrumental Analysis, Hokkaido University, for measurements of HR-ESI-MS. This work was partly supported by a Grant-in-Aid for Scientific Research from the Ministry of Education, Culture, Sports, Science and Technology of Japan.

\section{References}

1) Nahrstedt A., Butterweck V., Pharmacophychiatry, 30, 129-134 (1997).
2) Dostalek M., Pistovcakova J., Jurica J., Tomandl J., Linhart I., Sulcavá A., Hadasova E., Life Sci., 78, 239-244 (2005).

3) Medina M. A., Mrtínez-Proveda B., Amores-Sánchez M. I., Quesada A. R., Life Sci., 79, 105-111 (2006).

4) Beerhues L., Phytochemistry, 67, 2201-2207 (2006).

5) Tanaka N., Kubota T., Ishiyama H., Araki A., Kashiwada Y., Takaishi Y., Mikami Y., Kobayashi J., Bioorg. Med. Chem., 16, 5619-5623 (2008).

6) Tanaka N., Kubota T., Ishiyama H., Kashiwada Y., Takaishi Y., Ito J., Mikami Y., Shiro M., Kobayashi J., Heterocycles, 79, 917-924 (2008).

7) Tanaka N., Kakuguchi Y., Ishiyama H., Kubota T., Kobayashi J., Tetrahedron Lett., 50, 4747-4750 (2009).

8) Markham K. R., Ternai B., Stanley R., Geiger H., Mabry T. J., Tetrahedron, 34, 1389-1397 (1978).

9) Kasai R., Okihara M., Asakawa J., Mizutani K., Tanaka O., Tetrahedron, 35, 1427-1432 (1979). 